J. Lake Sci. (湖泊科学) , 2013, 25(1): 99-107

http: //www. jlakes. org. E-mail : jlakes@niglas.ac.cn

(C) 2013 by Journal of Lake Sciences

\title{
基于水声学方法的太湖鱼类空间分布和资源量评估
}

\author{
孙明波 $^{1,2}$, 谷孝鸿 ${ }^{1 * *}$, 曾庆飞 ${ }^{1}$,毛志刚 ${ }^{1,2}$, 谷先坤 ${ }^{1,2}$ \\ ( 1 : 中国科学院南京地理与湖泊研究所湖泊与环境国家重点实验室, 南京 210008) \\ (2:中国科学院大学,北京 100049)
}

\begin{abstract}
摘 要: 利用 BioSonics DT-X 科学回声探测仪 $(208 \mathrm{kHz}$ ) 在太湖 2011 年开捕前的 8 月对东部和北部湖区的鱼类进行了走 航式水声学调查,并结合地理信息系统 (GIS) 模型对调查湖区的鱼类大小组成、空间分布和资源量进行了评估. 结果表 明, 调查湖区的鱼类平均目标强度 (目标鱼类对声波的反射能力) 为 $-51.85 \pm 0.02 \mathrm{~dB}$, 平均体长在 $6 \mathrm{~cm}$ 左右, 体长范围 为 $2.35 \sim 89.33 \mathrm{~cm}$, 不同区域间的鱼类目标强度差异性显著, 表明不同区域间鱼类大小存在差异, 其中鱼类的最小平均 目标强度 $(-53.94 \pm 0.10 \mathrm{~dB})$ 出现在洞庭东西山之间, 最大平均目标强度 $(-50.27 \pm 0.14 \mathrm{~dB})$ 出现在光福湾. 调查湖区 的鱼类密度在 $0.43 \sim 3.90$ ind. $/ \mathrm{m}^{3}$ 之间, 采用地理信息系统 (GIS) 对调查湖区进行建模得到鱼类密度均值为 $2.27 \pm$ 0.57 ind. $/ \mathrm{m}^{3}$, 不同区域间鱼类密度差异性显著,鱼类密度在敞水区较高. 基于建模的栅格化数据评估调查湖区鱼类资源 量约为 $5.3 \times 10^{9} \mathrm{ind}$., 其中目标强度在 $-45 \mathrm{~dB}$ (体长约 $13 \mathrm{~cm}$ ) 以下的鱼类占 $98.49 \%$. 本文对水声学方法在大型浅水湖 泊中的应用进行了初步探索, 水声学方法可在一定程度上突破传统鱼类资源调查方法在较大空间尺度上的局限性,但在 调查时易受风浪、水生植物、船速的影响.
\end{abstract}

关键词: 水声学;太湖;鱼类;大小组成;空间分布;资源量

\section{Assessment of fish spatial distribution and biomass in Lake Taihu using hydroacoustic method}

\author{
SUN Mingbo ${ }^{1,2}$, GU Xiaohong ${ }^{1}$, ZENG Qingfei ${ }^{1}$, MAO Zhigang ${ }^{1,2}$ \& GU Xiankun ${ }^{1,2}$ \\ (1: State Key Laboratory of Lake Science and Environment, Nanjing Institute of Geography and Limnology, Chinese Academy \\ of Sciences, Nanjing 210008, P. R. China) \\ (2: University of Chinese Academy of Sciences, Beijing 100049, P. R. China)
}

\begin{abstract}
Before the fishing of Lake Taihu, a mobile hydroacoustic survey was performed in eastern and northern of Lake Taihu in August of 2011, with using BioSonics DT-X scientific echo-sounder (208 kHz). Combining Geographic Information System (GIS) model, then fish size component, spatial distribution and biomass are assessed. The results show that the mean target strength (acoustic reflectivity of target fish) in the surveying lake areas is $-51.85 \pm 0.02 \mathrm{~dB}$ and the mean length is about $6 \mathrm{~cm}$, ranging from 2.35 to $89.33 \mathrm{~cm}$. The fish target strength in different areas is significantly different, which means there is significant difference of fish size, with the minimum mean target strength $(-53.94 \pm 0.10 \mathrm{~dB})$ in the middle of Dongshan Island and Xishan Island and the maximum mean target strength $(-50.27 \pm 0.14 \mathrm{~dB})$ in Guangfu Bay. The mean fish density is $2.27 \pm 0.57 \mathrm{ind} . / \mathrm{m}^{3}$, ranging from 0.43 to 3.90 ind. $/ \mathrm{m}^{3}$ in all survey areas, where a high fish density occurred in open water zone. Based on the raster data gained from fish distribution GIS model, the total amount of fish mantissa biomass are estimated to be $5.3 \times 10^{9}$ ind. , in which the fish with target strength lower than $-45 \mathrm{~dB}$ (length about $13 \mathrm{~cm}$ ) is $98.49 \%$. The present study provided a preliminary application of the hydroacoustic method and performed in big shallow lake for fish resources investigation. This can break through the limitations of the traditional investigation methods for fish resource assessment on large spatial scales, to a certain extent. The application is still influenced by stormy waves, aquatic plants and speed.
\end{abstract}

Keywords: Hydroacoustic; Lake Taihu; fish; size component; spatial distribution; biomass

* 国家科技支撑计划项目 (2012BAD25B06)、环保部环保公益项目 (2010467014) 和江苏省水产三项工程项目 (PJ2011-55) 联合资助. 2012-02-07 收稿;2012-07-18 收修改稿. 孙明波,男, 1987 年生, 硕士研究生; E-mail: sunmingbo2008@126.com.

** 通信作者;E-mail:xhgu@ niglas. ac. cn. 
随着仪器性能的提高和计算机软件的发展, 水声学方法已日益成为鱼类资源量评估的主要手段, 相对 于传统的方法, 水声学方法具有快速高效、调查区域广、不损害生物资源、提供连续数据、精确定位鱼类位 置、准确估算鱼类密度和资源量的优势, 目前已在国外普遍采用 ${ }^{[1-3]}$. 自 $1980 \mathrm{~s}$ 引人 “北斗” 号调查船的 SIMRAD EK500 回声探测仪, 水声学方法已较多应用于我国海洋渔业资源的调查和研究 ${ }^{[4-6]}$. 由于水深较浅, 水声 学方法在内陆水体中应用时受近声区、盲区、噪音、旁瓣效应、水生植物等影响较大,但随着水平探测技术、裂 波技术、时变增益、仪器性能、分析软件的发展与提高, 这些问题得到逐步解决, 国外学者也对内陆水体进行 了较多的相关研究 ${ }^{[7-9]} .21$ 世纪以来, 我国也陆续开展了水声学方法在内陆水体鱼类资源研究中的应 用 ${ }^{[10-14]}$, 主要有三峡水库、长江、珠江、青海湖、东湖等, 但对于浅水湖泊, 只见东湖进行了相关研究 ${ }^{[14-15]}$, 而太湖这 种大型浅水湖泊, 目前尚未见水声学方法的调查报道, 本文采用科学回声探测仪 BioSonics DT-X 进行应用探索.

太湖地处长江三角洲南缘, 气候温和、水系发达、水位稳定、湖底平坦、湖泊营养物质本底含量高、水生 生物种类丰富, 是典型的浅水富营养化湖泊, 具有发展湖泊渔业的良好环境 ${ }^{[16]}$. 根据记载, 太湖共有 107 种 鱼类,隶属于 14 目 25 科 73 属 ${ }^{[17]}$. 鱼类是太湖捕捞渔业的主体资源,占捕捞总量的 $85 \% \sim 95 \%$, 太湖捕捞渔 业总产量在 1952-2006 年的 55 a 间呈不断增长趋势, 从 1952 年的 $4060.7 \mathrm{t}$ 增加到 2006 年的 $32187 \mathrm{t}$, 其中 小型鱼类湖鲚为优势种, 产量占渔获物的比例从 1952 年的 $15.8 \%$ 增加至 2006 年的 $60.2 \%$, 而大中型鱼类 比例大幅度下降 ${ }^{[18]}$, 这与太湖的鱼类放流、富营养化、不合理的捕捞方式以及太湖改变的水力学特点有关. 为了保护太湖渔业的可持续发展, 太湖渔业管理会实施了封湖休渔的政策, 将太湖的禁渔期延长到 2 月 1 日 至 8 月 31 日, 建立大面积繁殖保护区, 制定了渔具渔法, 实施鱼类放流. 目前, 太湖的鱼类资源状况仅能通过 每年的渔获物产量来反映, 并不能完全代表实际的鱼类资源状况, 因此, 选择太湖开捕前的几天, 避开开捕 后捕捞作业船只和渔具对回声探测仪、鱼类自然空间分布的干扰, 采用水声学方法对太湖鱼类大小组成、空 间分布和资源量进行评估, 以期为太湖渔业生产和资源管理提供科学依据, 为制定一个更合理的捕捞强度 和捕捞方式提供有价值的参考,并对水声学方法在大型浅水湖泊中的应用进行探讨.

\section{1 研究区域与研究方法}

\section{1 研究地区}

太湖 $\left(30^{\circ} 55^{\prime} 40^{\prime \prime} \sim 31^{\circ} 32^{\prime} 58^{\prime \prime} \mathrm{N}, 119^{\circ} 52^{\prime} 32^{\prime \prime} \sim 120^{\circ} 36^{\prime} 10^{\prime \prime} \mathrm{E}\right.$ ) 位于长江三角洲南缘, 是我国五大淡水湖泊之

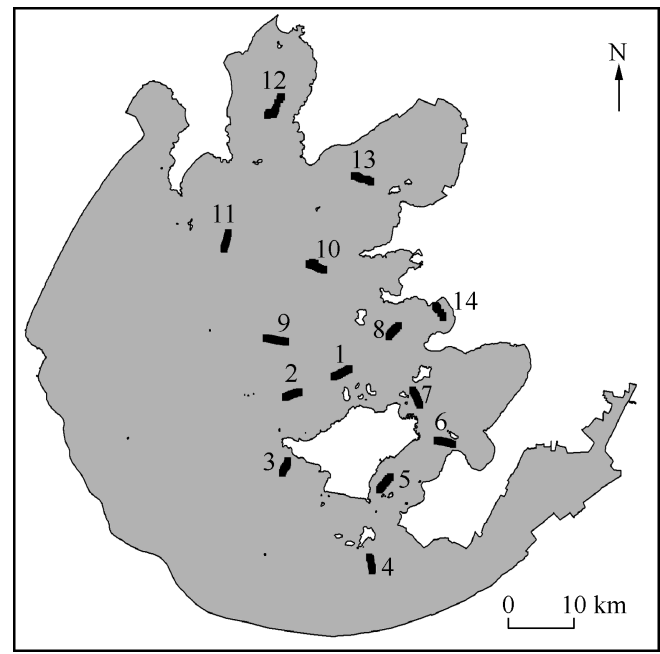

图 1 太湖东部和北部湖区的水声学调查区域 (图中数字代表调查区域, 根据调查先后划分, 一为航迹回放)

Fig. 1 Hydroacoustic survey area in eastern and northern Lake Taihu
一, 水位 $3.14 \mathrm{~m}$, 长 $68 \mathrm{~km}$, 最大宽 $56.0 \mathrm{~km}$, 平均宽 $35.7 \mathrm{~km}$, 水面面积为 $2338 \mathrm{~km}^{2}$, 最大水深 $3.3 \mathrm{~m}$, 平均 水深 $2.12 \mathrm{~m}$, 蓄水量 $5.14 \times 10^{9} \mathrm{~m}^{3}$, 湖底平坦. 最大风速 $24.7 \mathrm{~m} / \mathrm{s}$, 平均风速最大值介于 $3.8 \sim 4.3 \mathrm{~m} / \mathrm{s}$ 之间, 出 现在 3-5 月;最小值介于 $2.6 \sim 2.9 \mathrm{~m} / \mathrm{s}$, 出现在 8 月 或 12 月. 本次水声学调查在太湖的东部湖区和北部湖 区进行, 每个湖区各走航调查 7 个区域, 具体情况如

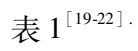

\section{2 调查研究方法}

2011 年太湖开捕前的 8 月 28-29 日, 采用水声学 方法对东部和北部湖区的鱼类空间分布和资源量进行 调查, 每天具体探测时间为 7:00-17:30. 使用太湖渔 政快艇 (85 马力) 进行走航, Garmin Oregon 450 导航仪 进行航线导航, 走航航速约为 $3 \mathrm{~m} / \mathrm{s}$, 区域 $1 \sim 14$ 的走 航航程和航线如表 1 和图 1 所示, 总长度约为 $38 \mathrm{~km}$.

本次水声学探测使用的仪器为 BioSonics DT-X 型 回声探测仪 $\left(6.5^{\circ}\right.$ 分裂波束数字换能器, 工作频率为 $208 \mathrm{kHz}$ ). 太湖属于浅水湖泊, 采取水平式探测, 将换 能器用铁架固定于船舷, 人水深约 $0.3 \mathrm{~m}$, 波束与水面 
呈 $15^{\circ}$ 倾斜,波束在 $8 \mathrm{~m}$ 左右的时候可以探测到水底. 利用 BioSonics Acquisition 6.0 软件进行水声学数据采 集, 采集过程中换能器脉冲频率为 $4 \mathrm{pps}$, 脉冲宽度为 $0.5 \mathrm{~ms}$, 数据收集阈值为 $-130 \mathrm{~dB}$, 数据收集距离为 $1 \sim$ $20 \mathrm{~m}$, 采用 Garmin GPS 17x HVS 对 GPS 数据同步采集存储. 实测水温为 $29.6^{\circ} \mathrm{C}$, 使用 $36 \mathrm{~mm}$ 的碳化铇标准 球对仪器进行实地校准.

表 1 太湖东部湖区和北部湖区水声学调查的基本信息

Tab. 1 The basic information of hydroacoustic survey in eastern and northern Lake Taihu

\begin{tabular}{|c|c|c|c|c|c|}
\hline 调查湖区 & 湖区环境特征 & 走航区域 & 走航航程/ km & 平均水深/m & 区域位置 \\
\hline \multirow[t]{7}{*}{ 东部湖区 } & $\begin{array}{l}\text { 水生植物分布广泛,生物多样性较 } \\
\text { 高, 水质较好, 为草型湖区 }\end{array}$ & 1 & 2.7 & 2.18 & $\begin{array}{l}\text { 西山岛北侧与敞水区 } \\
\text { 的交界处,横山外围 }\end{array}$ \\
\hline & & 2 & 2.4 & 2.32 & $\begin{array}{l}\text { 西山岛北侧与敞水区 } \\
\text { 的交界处,竹山东侧 }\end{array}$ \\
\hline & & 3 & 2.2 & 2.09 & $\begin{array}{l}\text { 西山岛西侧与敞水区 } \\
\text { 的交界处 }\end{array}$ \\
\hline & & 4 & 2.3 & 2.12 & $\begin{array}{l}\text { 东西太湖的交汇处, } \\
\text { 为生态多样性保护区 }\end{array}$ \\
\hline & & 5 & 2.6 & 2.11 & $\begin{array}{l}\text { 洞庭东西山间, 为鱼 } \\
\text { 类繁殖保护区 }\end{array}$ \\
\hline & & 6 & 2.7 & 1.95 & $\begin{array}{l}\text { 余山岛西侧, 为鱼类 } \\
\text { 繁殖保护区 }\end{array}$ \\
\hline & & 7 & 2.6 & 2.41 & $\begin{array}{l}\text { 西山大桥附近, 为鱼 } \\
\text { 类繁殖保护区 }\end{array}$ \\
\hline \multirow[t]{7}{*}{ 北部湖区 } & $\begin{array}{l}\text { 以高生物量的浮游植物和频发的 } \\
\text { 蓝藻水华为明显特征, 水质较差, } \\
\text { 水生植被消亡, 为藻型湖区 }\end{array}$ & 8 & 2.3 & 2.37 & $\begin{array}{l}\text { 沿岸带, 漫山岛东南 } \\
\text { 侧,为螺蚬保护区 }\end{array}$ \\
\hline & & 9 & 3.1 & 2.51 & 湖心敞水区 \\
\hline & & 10 & 3.0 & 2.78 & 湖心敞水区 \\
\hline & & 11 & 3.1 & 2.34 & 湖心敞水区 \\
\hline & & 12 & 3.5 & 2.17 & $\begin{array}{l}\text { 梅梁湾, 定置渔具禁 } \\
\text { 止捕捞区 }\end{array}$ \\
\hline & & 13 & 3.3 & 2.03 & 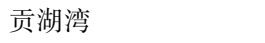 \\
\hline & & 14 & 2.2 & 2.64 & $\begin{array}{l}\text { 沿岸带, 为螺蚬保护 } \\
\text { 区、禁渔区、 }\end{array}$ \\
\hline
\end{tabular}

\section{3 渔获物采样调查}

2011 年 9 月 6 日对高踏网渔获物随机抽样调查, 高踏网由 2 只渔船形成作业组,网的长度 $2000 \mathrm{~m}$ 左右, 网目 $1 \mathrm{~mm}$, 机械收网, 可将围网范围内的鱼一网打尽. 共对 3 个作业组的渔获物进行随机取样, 统计记录鱼 类种类、尾数比例、体长、体重数据.

\section{4 数据处理及分析}

1.4.1 水声学数据处理及分析 对采集到的水声学数据用 BioSonics Visual Analyzer 4.1 进行分析,将每个航 段平均分成 10 个相等的单元,记录每个单元的中心坐标,数据分析从波束的 $2 \mathrm{~m}$ 处开始,同步输出单体回声 识别结果, 单体回声识别参数为: 回波阈值为 $-60 \mathrm{~dB}$, 相关系数为 0.90 , 最小脉冲宽度系数为 0.75 , 最大脉 冲宽度系数为 3 , 终止脉冲宽度为 $-12 \mathrm{~dB}$, 时变增益 $(\mathrm{TVG})$ 为 $40 \operatorname{lgR}$. 采用 $\mathrm{Misund}^{[23]}$ 的方法计算鱼类密度, 将单体回声计数结果除以探测体积,得到各单元的鱼类密度,计算公式为:

$$
V=\frac{1}{3} \pi \cdot \tan \frac{\theta}{2} \cdot\left(R^{3}-r^{3}\right)
$$




$$
\rho=N / \sum_{i=p}^{i=1} V_{i}
$$

式中, $V$ 为单个脉冲波束探测的水体体积 $\left(\mathrm{m}^{3}\right), \theta$ 为回声探测仪的角度 $6.5^{\circ}, r$ 为探测波束数据分析的起始 距离 $(\mathrm{m}), R$ 为探测波束数据分析的终止距离 $(\mathrm{m}), \rho$ 为鱼类密度 $\left(\right.$ ind.$\left./ \mathrm{m}^{3}\right), N$ 为回声计数得到鱼类回声信 号个数, $p$ 为单元总的脉冲数, $i$ 为单元内的第 $i$ 个脉冲.

将各单元所有单体回声结果根据 $T S$ (目标强度) 分为 $-60 \sim-55 、-55 \sim-50 、-50 \sim-45 、-45 \sim$ $-40 、-40 \sim-35 、-35 \sim-30 \mathrm{~dB}$ 六段, 分别统计各 TS 段的单体回声数, 同样利用上述方法计算各单元分 $T S$ 段的鱼类密度和平均目标强度. 本文根据 Foote ${ }^{[24]}$ 经验公式对鱼类长度进行估算: $T S=20 \lg L-67.4$, 式 中, $T S(\mathrm{~dB})$ 为鱼类的目标强度, $L(\mathrm{~cm})$ 为目标鱼体的体长.

1.4 .2 鱼类空间分布建模及资源量评估 对于不同探测区域的鱼类空间分布特征, 采用 ArcGIS 10.0 软件进 行空间分布的建模 ${ }^{[11]}$. 将计算出的各单元鱼类密度、各区域的均水深, 各单元航段中心坐标数据导人 ArcGIS 平台, 采用 IDW 方法进行栅格插值运算 ${ }^{[25-26]}$. 设定栅格大小为 $0.0026^{\circ}$, 约为 $0.29 \mathrm{~km} \times 0.25 \mathrm{~km}$, 设定幂 值 $p=2$, 在面积为 $1194.8 \mathrm{~km}^{2}$ 的东部和北部湖区中共得到 1600 多个栅格, 导出每个栅格鱼类密度和水深的 数值. 利用导出的每个栅格鱼类密度的数值、水深数值和栅格面积进行乘积计算得到各栅格的鱼类尾数, 最 后统计所有栅格的鱼类总尾数, 从而获得鱼类的资源总量, 计算公式为:

$$
B=\sum_{i=1}^{k} \rho_{i} \cdot S_{i} \cdot h_{i}
$$

式中, $B$ 为鱼类资源总尾数 (ind.), $\rho_{i}$ 为每个栅格的鱼类密度值 (ind. $/ \mathrm{m}^{3}$ ), $k$ 为所有栅格数, $S_{i}$ 为栅格的面积 $\left(\mathrm{m}^{2}\right), h_{i}$ 为栅格水深 $(\mathrm{m})$. 利用同样的方法对上述各 TS 段的鱼类空间分布进行建模, 并评估相应 TS 段的资 源尾数.

1.4 .3 数据的统计分析 本文所进行的回声信号 TS 值分布和鱼类密度分布的正态性检验、回声信号 TS 值 差异性非参数检验、鱼类密度的描述性统计, 鱼类密度分布方差齐性检验、鱼类密度分布单因素方差分析、 鱼类密度和目标强度的区域聚类分析均采用 SPSS 17.0. 鱼类密度变异系数 $C V=S / X$ (式中, $S$ 为标准差 (ind. $/ \mathrm{m}^{3}$ ), $X$ 为平均值 $\left(\right.$ ind. $\left./ \mathrm{m}^{3}\right)$ ). 鱼类分布建模采用 ArcGIS 10.0.

\section{2 结果和分析}

\section{1 渔获物组成}

从高踏网随机取样, 在三个高踏网作业组共采集鱼类 $17.19 \mathrm{~kg}$, 包括 8 种鱼类, 尾数比例为: 湖鲚(Coilia ectenes taihuensis) 92. 86\% 、银鱼 5.51\%、间下鱵( Hyporhamphus intermedius) $0.65 \%$ 、鲫 ( Carassius auratus) 0.43\%、鲤(Cyprinus carpio)0.25\%、黄颡鱼(Pelteobagrus fulvidraco)0.20\%、鳙(Aristichthys nobilis) $0.05 \%$ 、鲢 (Hypophthalmichthys molitrix) $0.02 \%$. 鱼类体长与体重统计结果如表 2 , 其中湖鲚、银鱼、间下鰔随机各取 20 尾,其他鱼类个体数较少从渔获物随机取样补齐至 20 尾.

表 2 渔获物调查

\begin{tabular}{|c|c|c|c|c|c|c|}
\hline \multirow{2}{*}{ 种类 } & \multicolumn{3}{|c|}{ 体长 $/ \mathrm{cm}$} & \multicolumn{3}{|c|}{ 体重/g } \\
\hline & 最小值 & 平均值 \pm 标准差 & 最大值 & 最小值 & 平均值 \pm 标准差 & 最大值 \\
\hline 湖鲚 & 5.0 & $8.4 \pm 2.4$ & 11.8 & 0.92 & $2.74 \pm 1.27$ & 5.86 \\
\hline 银鱼 & 7.5 & $8.3 \pm 0.4$ & 9.3 & 1.71 & $2.33 \pm 0.29$ & 2.81 \\
\hline 鲫 & 9.5 & $15.0 \pm 2.3$ & 20.4 & 23.40 & $108.10 \pm 8.52$ & 246.20 \\
\hline 间下鱵 & 9.4 & $11.2 \pm 0.7$ & 12.6 & 1.45 & $2.65 \pm 0.41$ & 3.48 \\
\hline 鲤 & 18.3 & $21.5 \pm 2.0$ & 26.0 & 155.20 & $233.75 \pm 58.33$ & 398.11 \\
\hline 鳙 & 20.8 & $41.1 \pm 7.1$ & 60.2 & 163.02 & $1474.64 \pm 740.49$ & 4017.93 \\
\hline 鲢 & 23.0 & $37.9 \pm 8.5$ & 60.0 & 219.82 & $1279.01 \pm 858.07$ & 3895.12 \\
\hline 黄颡鱼 & 12.0 & $15.8 \pm 1.1$ & 18.1 & 74.00 & $63.98 \pm 8.52$ & 32.40 \\
\hline
\end{tabular}

Tab. 2 Survey of fish catch 


\section{2 鱼类大小组成特征}

在本次所有走航调查航段内共收集到 88121 个回声信号, 平均 $T S$ 值为 $-51.85 \pm 0.02 \mathrm{~dB}$, 其中最大 $T S$ 值为 $-28.38 \mathrm{~dB}$, 最小 $T S$ 值为 $-59.99 \mathrm{~dB}$ (回波阈值). 按照 Foote 经验公式推算鱼类平均体长约为 $6 \mathrm{~cm}$, 体 长范围为 $2.35 \sim 89.33 \mathrm{~cm}$, 对各 $T S$ 段 (从 小到大) 进行统计, 相应平均体长约为 3 、 $5 、 9 、 17 、 30$ 和 $51 \mathrm{~cm}$. 在本次调查中, 探测 到的大于 $-30 \mathrm{~dB}$ 的目标信号 9 个, 即体 长大于 $74 \mathrm{~cm}$ 的鱼非常少, 在沿岸带的区 域 14 中探测到 2 个, 在东西太湖交汇处 ( 区域 4) 中探测到 3 个,其余 4 个在敞水 的藻型湖区 (区域 $9 、 10 、 11 、 12$ ) 探测到, 目标个体数非常低,故不再分 $T S$ 段进行 统计. 在敞水区的区域 10 中探测到的回 声信号最多,在沿岸带的区域 14 中探测 的回声信号最少, 各调查区域的回波数如

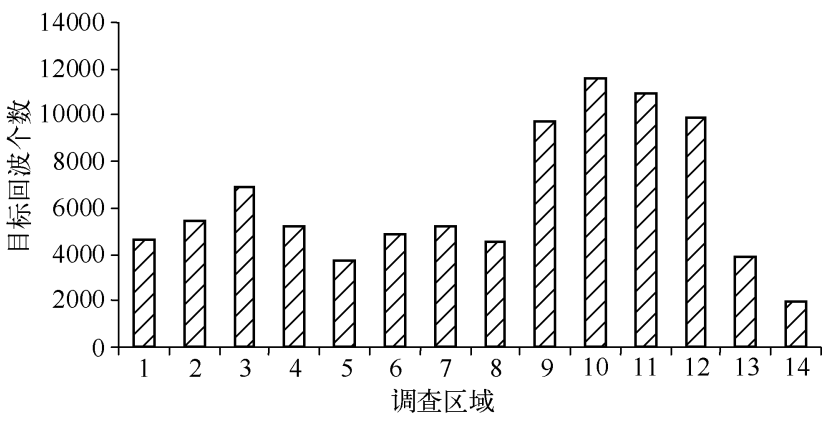

图 2 各调查区域目标回波数

Fig. 2 The target echo number of every survey area 图 2 所示.

经 Shapiro-Wilk 正态性检验, 走航的 14 个调查区域中所探测到的鱼类回声信号 $T S$ 值并不符合正态分 布 $(P<0.05)$, 各调查区域中低 $T S$ 值的鱼类个体占有较高的比例 (图 3). 故利用非参数检验方法对 14 个调 查区域内鱼类回声信号 $T S$ 值差异性进行比较, 发现 14 个调查区域内的鱼类回声信号 $T S$ 值差异显著, 即在 不同区域里鱼类的大小差异显著 $(P<0.05)$, 洞庭东西山间 ( 区域 5) 的平均 $T S$ 值最小 $-53.94 \pm 0.10 \mathrm{~dB}$, 沿岸带的区域 14 平均 $T S$ 值最大 $-50.27 \pm 0.14 \mathrm{~dB}$, 各走航调查区域平均 $T S$ 见图 4.

计算各调查区域内不同 TS 值段的鱼类回声信号所占的比例表明所有调查区域中, TS 值在 - 55 $-50 \mathrm{~dB}$ 段的鱼类回声信号所占的比例都是最高的,除洞庭东西山间的区域 5 为 $49.36 \%$, 其他都在 $50 \%$ 以 上(图 3). 各区域中 $-45 \sim-40 、-40 \sim-35 、-35 \sim-30 \mathrm{~dB}$ 三个 TS 值段的鱼类回声信号所占的比例都非 常低. 洞庭东西山间的区域 5 中, $T S$ 值在 $-60 \sim-55 \mathrm{~dB}$ 段的鱼类回声信号所占比例有所上升, 而 $T S$ 值段 $-55 \sim-50 \mathrm{~dB}$ 与 $-50 \sim-45 \mathrm{~dB}$ 的鱼类回声信号所占比例有所下降. 同位于敞水区的区域 9、10、11 和沿岸 带的区域 8 四个区域的鱼类回声信号 TS 值比例分布相似. 在沿岸带的区域 14 中, $-40 \sim-35 \mathrm{~dB}$ 与 $-35 \sim$ $-30 \mathrm{~dB}$ 两个 TS 值段的鱼类回声信号所占比例都有所上升.

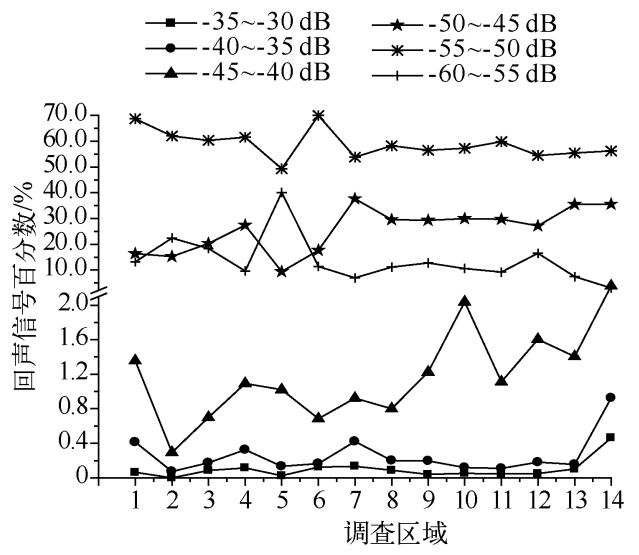

图 3 各调查区域不同 $T S$ 段的鱼类回声信号百分比

Fig. 3 The proportion of fish echo signal in different $T S$ in every survey area

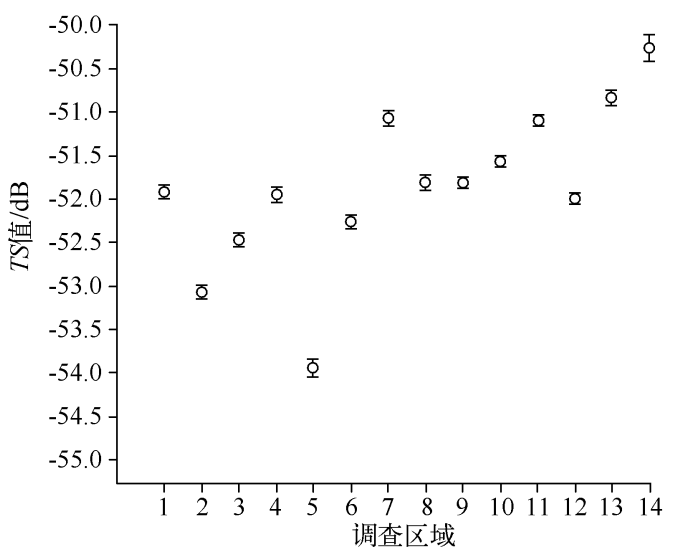

图 4 各调查区域回声信号 $T S$ 均值

Fig. 4 The average $T S$ of echo in every survey area 


\section{3 不同区域鱼类密度}

在 14 个区域的各单元航段中, 密度最小值为 $0.43 \mathrm{ind} . / \mathrm{m}^{3}$, 位于西山大桥附近的区域 $7\left(31^{\circ} 10^{\prime} \mathrm{N}\right.$, $\left.120^{\circ} 21^{\prime} \mathrm{E}\right)$; 密度最大值为 $3.9 \mathrm{ind} . / \mathrm{m}^{3}$, 位于湖心北端的敞水区区域 $11\left(31^{\circ} 19^{\prime} \mathrm{N}, 120^{\circ} 8^{\prime} \mathrm{E}\right)$. 在走航调查的所有 区域中, 敞水区的区域 11 均密度最大, 为 $3.67 \pm 0.12 \mathrm{ind} . / \mathrm{m}^{3}$, 沿岸区的区域 14 的均密度最小, 为 $0.58 \pm$ 0.04 ind. $/ \mathrm{m}^{3}$ (表 3 ). 经方差齐性检验, 14 个调查区域中鱼类密度分布方差不具备同质性 $(P<0.05)$, 这表 明在不同调查区域中, 鱼群密度空间分布不同. 对各个调查区域内鱼类密度的变异系数比较, 洞庭东西山间 的区域 5 变异系数最大为 0.35 , 敞水区的区域 10 变异系数最小为 0.04 , 说明在洞庭东西山间鱼类分布比较 不均匀, 而在敞水区内鱼类密度分布相对均匀, 所有区域的鱼类密度变异系数都小于 1 , 根据孙儒泳种群分 布型的划分标准 ${ }^{[27]}$, 调查区域的鱼类都属于均匀分布, 无成群存在. 对各调查区域的鱼类密度在未假定方差 齐性下采用 Games-Howell 方法进行多重比较 (表 3).

表 3 不同调查区域鱼类密度差异性比较

Tab. 3 Comparison of fish density differences in different survey areas

\begin{tabular}{|c|c|c|c|c|c|c|c|c|c|c|c|c|c|c|}
\hline 调查区域 & 2 & 3 & 4 & 5 & 6 & 7 & 8 & 9 & 10 & 11 & 12 & 13 & 14 & 鱼类密度 $/\left(\right.$ ind. $\left./ \mathrm{m}^{3}\right)$ \\
\hline 2 & - & & & & & & & & & & & & & $2.56 \pm 0.46$ \\
\hline 3 & - & & & & & & & & & & & & & $2.06 \pm 0.22$ \\
\hline 4 & - & - & & & & & & & & & & & & $2.19 \pm 0.15$ \\
\hline 5 & $*$ & $*$ & $*$ & & & & & & & & & & & $1.04 \pm 0.26$ \\
\hline 6 & - & * & * & $*$ & & & & & & & & & & $2.85 \pm 0.20$ \\
\hline 7 & $*$ & - & * & - & $*$ & & & & & & & & & $1.46 \pm 0.30$ \\
\hline 8 & * & * & * & - & * & - & & & & & & & & $1.34 \pm 0.25$ \\
\hline 9 & - & * & * & $*$ & - & * & * & & & & & & & $2.89 \pm 0.15$ \\
\hline 10 & - & $*$ & * & $*$ & $*$ & * & * & $*$ & & & & & & $3.44 \pm 0.10$ \\
\hline 11 & $*$ & * & * & $*$ & * & * & * & $*$ & - & & & & & $3.67 \pm 0.12$ \\
\hline 12 & - & * & $*$ & $*$ & - & $*$ & $*$ & - & - & - & & & & $2.93 \pm 0.33$ \\
\hline 13 & $*$ & $*$ & * & - & $*$ & - & - & $*$ & $*$ & $*$ & $*$ & & & $1.35 \pm 0.05$ \\
\hline 14 & $*$ & * & * & - & $*$ & * & * & $*$ & $*$ & $*$ & $*$ & - & & $0.58 \pm 0.04$ \\
\hline 1 & $*$ & $*$ & * & - & $*$ & - & - & $*$ & $*$ & $*$ & * & - & $*$ & $1.31 \pm 0.21$ \\
\hline
\end{tabular}

- 表示在 $P=0.05$ 不显著, $*$ 表示在 $P=0.05$ 显著.

选取各区域的鱼类密度、TS、TS 变异系数作为变量, 采用质心聚类法对各区域进行系统聚类分析, 同一 类在一定程度上体现区域间的鱼类密度和大小组成两者的相似性, 可分为五类: 区域 $1 、 8 、 3$ 和 4 为 I 类, 区 域 2 为 III类, 区域 6、9、10、11 和 12 为 III 类, 区域 7、13 和 14 为 $\mathrm{IV}$ 类, 区域 5 为 $\mathrm{V}$ 类 (图 5). 同一类也反映出 了环境的相似性, 如 I 类主要为东部湖区水草较多的区域, III类中的区域主要为敞水区, 而 IV 类中的 3 个区 域鱼类密度较低.

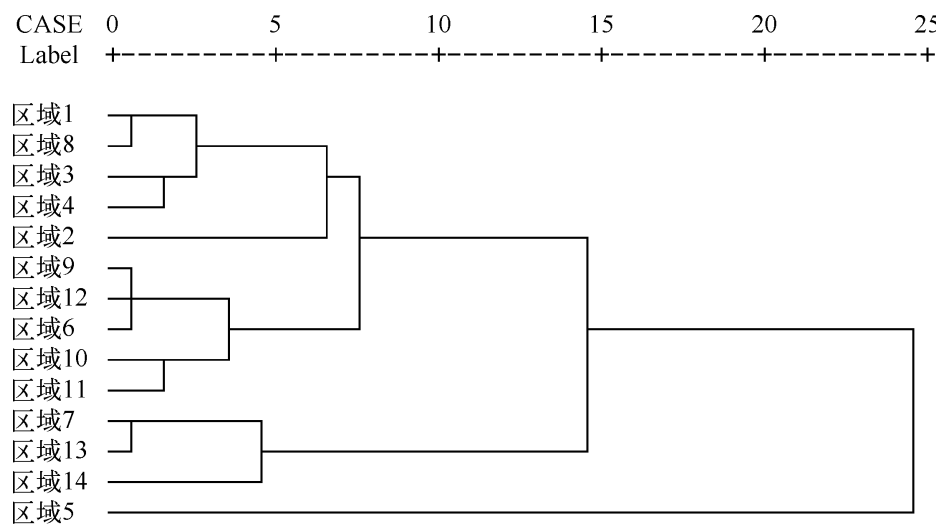

图 5 调查区域聚类图

Fig. 5 Cluster dendrogram of survey areas 


\section{4 鱼类空间分布建模和资源总量评估}

对面积为 $1194.8 \mathrm{~km}^{2}$ 的东部和北部湖区进行地理信 息系统 (GIS) 建模 (图 6), 在图示的湖区中鱼类密度建模 均值为 $2.27 \pm 0.57 \mathrm{ind} . / \mathrm{m}^{3}$, 初步估算鱼类资源总尾数为 $5.3 \times 10^{9}$ ind., 其中 $-60 \sim-55 、-55 \sim-50 、-50 \sim-45$ 、 $-45 \sim-40 、-40 \sim-35 、-35 \sim-30 \mathrm{~dB}$ 的鱼类资源尾数 分别为 $6.9 \times 10^{8} 、 3.1 \times 10^{9} 、 1.4 \times 10^{9} 、 6.6 \times 10^{7} 、 1.0 \times 10^{7}$ 、 $3.7 \times 10^{7} \mathrm{ind}$, 所占总尾数的百分比分别为 $13.10 \%$ 、 $58.94 \% 、 26.45 \% 、 1.25 \% 、 0.19 \% 、 0.07 \%$, 小于 $-45 \mathrm{~dB}$ (约 $13 \mathrm{~cm}$ ) 的鱼类所占的尾数比例达到了 $98.49 \%$. 结合渔 获物和根据 Foote 公式得到的平均体长值赋予各 TS 段 (从 小到大) 的鱼类平均体重约为 $0.8 、 1 、 4 、 20 、 280$ 和 $1250 \mathrm{~g}$, 并乘以相应各 $T S$ 段的鱼类资源尾数得到资源重量, 对各 $T S$ 段的资源重量相加初步估算调查湖区鱼类资源总重量 约为 $1.8 \times 10^{4} \mathrm{t}$.

\section{3 讨论}

本文在太湖开捕前期利用水声学方法和地理信息系

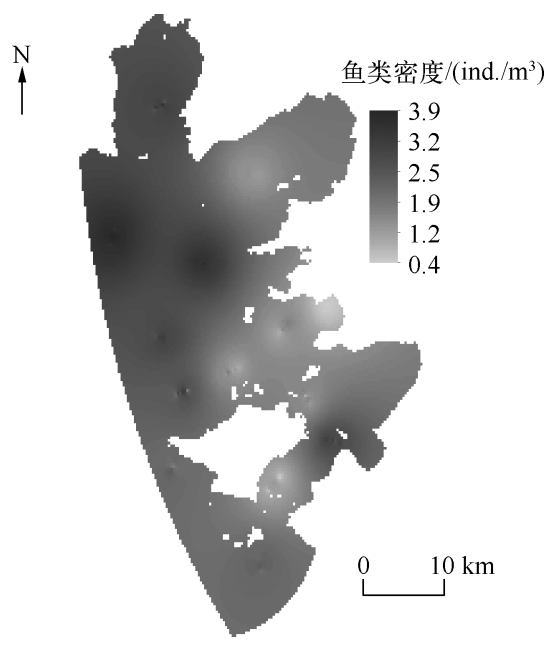

图 6 太湖东部和北部湖区开捕前 鱼类密度分布

Fig. 6 The fish density distribution of northern and eastern Lake Taihu before fishing 统 (GIS) 模型对太湖鱼类大小组成特征、鱼类空间分布和 鱼类资源量进行了调查研究, 但在实际的调查中仍遇到了一些问题. 风力在 $3 \sim 4 \mathrm{~m} / \mathrm{s}$ 以上时, 波浪较大, 采 用回声探测仪进行水平探测受到的影响非常大 ${ }^{[28]}$, 太湖属于大型浅水湖泊, 需进行水平探测, 本次调查选在 风浪较小的 9 月 28 日和 29 日进行, 走航航速在 $8 \mathrm{~km} / \mathrm{h}$ 左右, 而在风浪较大的敞水区船速须在 $5 \mathrm{~km} / \mathrm{h}$ 以 下, 将风浪的影响尽可能最小化. 另外在走航的一些区域中,水草生长较多,对鱼类的探测会造成较大影响, 后期的分析中对这些区域进行了去除,在水草较多的浅水水体,利用水声学方法对鱼类资源量调查并不适 合, Hughes 在泰晤士河的研究中也提出了沉水植物较多时走航式水声学调查并不理想, 但对于泰晤士河鱼 类资源量评估和分布研究仍是一个高效的方法 ${ }^{[7]}$. 在探测的区域中没有发现明显的鱼类聚群现象, 个体间 相对分散,采用回声计数的方法进行密度计算更为合适 ${ }^{[10]}$.

鱼类回声信号 TS 值依赖于鱼类大小、脉冲发射频率、鱼类在波束中的位置 ${ }^{[29]}$ 、游泳动作 ${ }^{[30]}$ 和环境的物 理条件 ${ }^{[31]}$, 超声波在浅水湖泊水平扩散与垂直扩散有所不同, TVG 也有所不同 ${ }^{[32]}$, 仍缺少在浅水水体中水 平探测时回声信号 $T S$ 值与鱼类大小关系的研究, 尚无法准确根据鱼类 $T S$ 值计算出相应的鱼类大小, 对于 鱼类大小的初步估计借鉴国外的一些垂直探测的经验公式,不同的研究应以不同 TS 值下的密度进行准确 比较. 尽管如此, 水声学方法仍是鱼类资源量统计的较为全面的方法, 在调查过程中克服了其他传统方法工 作效率低、工作量大、难以在得到大空间尺度下的鱼类密度、无法准确获得鱼类自然分布状态下连续数据等 局限性 ${ }^{[10,33-34]}$, 可以准确的给出鱼类大小组成特征、空间分布和尾数资源量情况.

本次调查所有探测到的鱼类回声信号平均 $T S$ 值为 $-51.85 \pm 0.02 \mathrm{~dB}$, 根据 Foote 的 $T S$ 值与体长经验公 式估算体长大约在 $6 \mathrm{~cm}$, 本文在 9 月 6 日进行了高踏网渔获物采样统计, 高踏网基本上可以捕捞到所有鱼 类, 其中尾数占绝大部分的湖鲚 $(92.86 \%)$ 的平均体长为 $8.38 \pm 2.41 \mathrm{~cm}$, 两者比较可见通过水声学方法调 查得到的平均 $T S$ 值按照 Foote 经验公式计算出的相应平均体长偏低,但仍在渔获物平均体长的 $95 \%$ 置信度 上下限区间内. 渔获物统计结果亦表明小型鱼类尾数比例占据了 $99 \%$ 以上,水声学方法调查结果与之具有 一致性, 这也与其他学者太湖鱼类小型化的研究结论相符合 ${ }^{[35]}$, 太湖的主要优势种为湖魰, 其种群为小型鱼 类,一般不会超过 $20 \mathrm{~cm}$, 体长多在 $10 \mathrm{~cm}$ 以下. 本次调查所探测到的回声信号中较大的 $T S$ 值 $(>-30 \mathrm{~dB})$ 非常少, 分布在调查区域 $4 、 9 、 10 、 11 、 12$ 和 14 , 区域 4 处于东西太湖交汇处的草型湖区, 为生物多样性保护 区, 可能是较大的草鱼或鲤; 区域 $9 、 10$ 和 11 在湖心敞水区, 区域 12 在梅梁湾,都属于典型的藻型湖区, 可能 是较大的鲢鳙; 而区域 14 位于螺蚬保护区和太湖渔业实验站保护区, 常年禁渔,亦有可能存在较大个体 
鱼类 ${ }^{[20]}$.

在所有调查区域内, 鱼类密度最大的为敞水区的区域 $11\left(3.67 \pm 0.12 \mathrm{ind} . / \mathrm{m}^{3}\right)$, 最小的为沿岸区的区 域 $14\left(0.58 \pm 0.04 \mathrm{ind} . / \mathrm{m}^{3}\right)$, 这可能与区域 11 属于敞水区有很大关系, 太湖中的小型鱼类湖鲚、银鱼在敞水 区分布较多 ${ }^{[36-37]}$, 聚类分析中也体现出敞水区探测区域 $9 、 10 、 11$ 和 12 的密度具有一致性,而沿岸区的区域 14 中虽然密度较低, 但是较大个体的鱼类 TS 比例却有所上升, 这可能是由于该区域属于保护区受到常年的 禁渔保护有关. 各个调查区域的鱼类密度差异性显著, 根据地理信息系统建模的分布图显示, 鱼类密度在敞 水区较高, 在西山岛外围的横山西北侧相对较低, 东西山间的东北侧的鱼类密度要略高于西南侧, 另外可以 明显发现梅梁湾的鱼类密度明显高于贡山湾的鱼类密度, 鱼类密度的分布与流速、植被状况、浮游生物、底 质特点、保护区建立、湖泊营养化水平等因素有关 ${ }^{[3840]}$, 对于太湖鱼类的分布与这些因子的关系需要进一步 的探索研究.

\section{4 参考文献}

[ 1 ] Jurvelius J, Leinikki J, Mamylov V et al. Stock assessment of pelagic three-spined stickleback (Gasterosteus aculeatus) : A simultaneous up-looking and down-looking echo-sounder study. Fisheries Research, 1996, 27 (4) : 227-241.

[ 2 ] Stockwell JD, Yule DL, Hrabik TR et al. Vertical distribution of fish biomass in lake superior: Implications for day bottom trawl surveys. North American Journal of Fisheries Management, 2007, 27 (3) : 735-749.

[ 3 ] Ohshimo S, Mitani T, Honda S. Acoustic surveys of spawning Japanese sardine, Sardinops melanostictus, in the waters off western and southern Kyushu, Japan. Fisheries Science, 1998, 64(5) : 665-672.

[4] 唐启升,王为祥,陈毓桢等. 北太平洋狭鳕资源声学评估调查研究. 水产学报, 1995,19(1):8-20.

[5]陈国宝,李永振, 赵宪勇等. 南海北部海域重要经济鱼类资源声学评估. 中国水产科学,2005,12(4):445-451.

[6]李永振, 陈国宝, 赵宪勇等. 南海北部海域小型非经济鱼类资源声学评估. 中国海洋大学学报, 2005,35 (2): 206-212.

[ 7 ] Hughes S. A mobile horizontal hydroacoustic fisheries survey of the River Thames, United Kingdom. Fisheries Research, $1998,35(1 / 2)$ : 91-97.

[ 8 ] Knudsen FR, Saegrov H. Benefits from horizontal beaming during acoustic survey: application to three Norwegian lakes. Fisheries Research, 2002, 56(2) : 205-211.

[ 9 ] Drastik V, Kubecka J, Cech M et al. Hydroacoustic estimates of fish stocks in temperate reservoirs: day or night surveys? Aquatic Living Resources, 2009, 22(1): 69-77.

[10]谭细畅, 史建全, 张 宏等. EY60 回声探测仪在青海湖鱼类资源量评估中的应用. 湖泊科学, 2009, 21 (6) : $865-872$.

[11] 陶江平, 艾为明, 龚昱田等. 采用渔业声学方法和 GIS 模型对楠溪江鱼类资源量及空间分布的评估. 生态学报, $2010, \mathbf{3 0}$ ( 11 ) :2992-3000.

[12］张慧杰,杨德国,危起伟等. 葛洲坝至古老背江段鱼类的水声学调查. 长江流域资源与环境,2007,16(1):86-91.

[13] 陶江平,陈永柏,乔 晔等. 三峡水库成库期间鱼类空间分布的水声学研究. 水生态学杂志, 2008,28(5):25-33.

[14] 立川贤一,朱志荣,三浦泰藏. 用浅水湖泊型鱼探仪估算东湖鱼群数量. 水生生物学报, 1986,10(4):311-326.

[15] 谭细畅,立川贤一. 东湖放养鱼类时空分布的水声学研究. 水生生物学报,2002,26(6) :585-590.

[16] 谷孝鸿, 朱松泉, 吴林坤等. 太湖自然渔业及其发展策略. 湖泊科学, 2009,21(1):94-100.

[17] 倪 勇,朱成德. 太湖鱼类志. 上海:上海科学技术出版社,2005:78-81.

[18］何 俊,谷孝鸿, 白秀玲. 太湖渔业产量和结构变化及其对水环境的影响. 海洋湖沼通报, 2009, (2):143-150.

[19］朱广伟. 太湖富营养化现状及原因分析. 湖泊科学,2008,20(1):21-26.

[20］谷孝鸿, 白秀玲, 江 南等. 太湖渔业发展及区域设置与功能定位. 生态学报,2006,26(7):2247-2254.

[21] 毛志刚, 谷孝鸿, 曾庆飞等. 太湖渔业资源现状 (2009-2010) 及与水体富营养化关系浅析. 湖泊科学, 2011,23 (6) : 967-973.

[22］王苏民,窦鸿身. 中国湖泊志. 北京: 科学出版社, 1998:261-268.

[23] Misund OA. Underwater acoustics in marine fisheries and fisheries research. Reviews in Fish Biology and Fisheries, 1997, $7(1): 1-34$.

[24] Foote KG. Fish target strengths for use in echo integrator surveys. Journal of the Acoustical Society of America, $1987, \mathbf{8 2}$ 
(3) : $981-987$.

[25] Petitgas P. Geostatistics for fish stock assessments : a review and an acoustic application. ICES Journal of Marine Science, $1993, \mathbf{5 0}(3)$ : 285-298.

[26] Li X, Cheng GD, Lu L. Comparison of spatial interpolation methods. Advance in Earth Sciences, 2000, 15 (3) : 260-265.

[27］孙儒泳. 动物生态学原理. 北京: 北京师范大学出版社, 1987:283-295.

[28] Knudsen FR, Saegrov H. Benefits from horizontal beaming during acoustic survey: application to three Norwegian lakes. Fisheries Research, 2002, 56(2) : 205-211.

[29] Kubecka J, Duncan A. Acoustic size vs. real size relationships for common species of riverine fish. Fisheries Research, $1998, \mathbf{3 5}(1 / 2): 115-125$.

[30 ] Dahl PH, Mathisen OA. Measurement of fish target strength and associated directivity at high frequencies. Journal of the Acoustical Society of America, 1983, 73(4) : 1205-1211.

[31] Ona E. Physiological factors causing natural variations in acoustic target strength of fish. Journal of the Marine Biological Association of the United Kingdom, 1990, 70(1): 107-127.

[32] Gerlotto F, Georgakarakos S, Eriksen PK. The application of multibeam sonar technology for quantitative estimates of fish density in shallow water acoustic surveys. Aquatic Living Resources, 2000, 13(5) : 385-393.

[33］詹秉义. 渔业资源评估. 北京:中国农业出版社,1995:236-253.

[34] 王 珂,段辛斌,刘绍平等.三峡库区大宁河鱼类的时空分布特征. 水生生物学报,2009,33(3):516-521.

[35] 朱松泉,刘正文,谷孝鸿. 太湖鱼类区系变化和渔获物分析. 湖泊科学,2007,19(6):664-669.

[36］邓思明,藏增嘉. 太湖敞水区鱼类群里结构特征和分析. 水产学报,1997,21(2):34-42.

[37］李圣法,藏增嘉. 太湖敞水区鱼类种间关系现状. 水产学报,1998,22(1):44-48.

[38] Vogler R, Milessi AC, Quinones RA. Influence of environmental variables on the distribution of Squatina guggenheim (Chondrichthyes, Squatinidae) in the Argentine-Uruguayan Common Fishing Zone. Fisheries Research, 2008, 91(2/3): 212-221.

[39] Tameishi H, Shinomiya H, Aoki I et al. Understanding Japanese sardine migrations using acoustic and other aids. ICES Journal of Marine Science, 1996, 53(2): 167-171.

[40] Godlewska M, Swierzowski A. Hydroacoustical parameters of fish in reservoirs with contrasting levels of eutrophication. Aquatic Living Resources, 2003, 16(3) : 167-173. 